\title{
Effects of letrozole co-treatment on outcomes of gonadotropin releasing hormone antagonist cycles in POSEIDON group 3 and 4 expected poor responders
}

Alper Kahraman ( $\square$ alper.k@hotmail.com )

Ankara University https://orcid.org/0000-0002-1689-2782

Firat Tulek

Memorial Atasehir Hospital

\section{Research Article}

Keywords: infertility, letrozole, live birth rate, poor responder, POSEIDON

Posted Date: February 2nd, 2022

DOI: https://doi.org/10.21203/rs.3.rs-1267736/v1

License: (9) This work is licensed under a Creative Commons Attribution 4.0 International License. Read Full License 


\section{Abstract}

Purpose: To evaluate the effects of adjuvant letrozole administration on outcomes of gonadotropin releasing hormone (GnRH) antagonist cycles with intracytoplasmic sperm injection in POSEIDON group 3 and 4 expected poor responder women.

Methods: This study was conducted by retrospective analysis of patients with expected poor ovarian response (POSEIDON group 3 and 4) that underwent $\mathrm{GnRH}$ antagonist cycles with intra-cytoplasmic sperm injection between 2010 and 2020. A total of 488 patients with letrozole co-administration and 2564 patients without any adjuvant treatment that underwent GnRH antagonist cycles within the selected period of time were included in the study.

Results: Implantation rates, clinical pregnancy rates and live birth delivery rates were significantly higher in letrozole administered patients in comparison to controls among POSEIDON group 3 women $(0.39 \pm 0.46$ vs $0.27 \pm 0.40, p=0.01 ; 46.1 \%$ vs $33 \%, p=0.014$; $42.7 \%$ vs $31.5 \%, p=0.034$, respectively). Mean total doses of gonadotropins required per cycle were significantly lower in letrozole administered patients among both POSEIDON group 3 and 4 women $(2864.65 \pm 878.47$ IU vs $3757.27 \pm 1088.89$ IU, $p<0.001$; $3286.06 \pm 770.16 \mathrm{IU}$ vs $3666.48 \pm 959.66 \mathrm{IU}, \mathrm{p}<0.001$, respectively).

Conclusion: Adjuvant letrozole co-administration in intracytoplasmic sperm injection cycles following GnRH antagonist protocol appears to improve implantation, clinical pregnancy and live birth delivery rates in women with POSEIDON group 3 expected poor ovarian response.

\section{Introduction}

Poor ovarian response (POR) is one of the major challenges in reproductive medicine that dramatically reduce the chance of success in assisted reproduction. For decades, several different criteria were used to define poor ovarian response until the definition suggested by ESHRE gained general acceptance and provide standardization for studies in literature. ESHRE defined poor ovarian response as $\leq 3$ ovarian follicles on the day of oocyte maturation triggering or $\leq 3$ oocytes obtained in a controlled ovarian stimulation cycle [1]. Particularly due to the heterogeneity in definition, prevalence of poor responder women ranges between $5.6 \%$ and $35.1 \%$ of in-vitro fertilization/embryo transfer cycles among studies in literature [2]. These patients have lower chance of conception and live birth rates are reported $6-23 \%$ in different studies $[3,4]$. Determining unequivocally effective management modalities for poor responder women have been problematic due to heterogeneity of underlying conditions and etiologies among poor responder women. Considering the various drawbacks of former classification systems, POSEIDON study group introduced the concept of "low-prognosis" and stratified these patients considering the common etiologic factors in each group with a pragmatic approach in determining management strategies and in attempt to standardize studies [5].

Several management modalities are suggested to increase IVF success rates in studies using older classification systems and diagnostic criteria for poor responders, including higher doses of gonadotropin administration, mild stimulation with using clomiphene citrate, reduced dose protocols, modified natural cycles, adjuvant agents and more radical interventions like in-vitro oocyte activation with limited success $[1,6]$. One of these modalities is adjuvant letrozole administration in controlled ovarian stimulation cycles.

Letrozole blocks conversation of androgens to estrogens by competitively inhibiting aromatase enzyme throughout the body that results with decreased estrogen and increased androgen levels [7]. Increase in androgen levels augments FSH activity and androgens are known to take crucial roles in pre-antral to early antral follicle transition [7]. Low androgen levels have been shown to be associated with impaired IVF success and transdermal testosterone pre-treatment has been demonstrated to increase live birth rates in poor responder women in previous studies [8, 9]. Unlike clomiphene citrate that depletes estrogen receptors, letrozole left negative estrogen feed-back mechanisms intact, therefore interferes less in the processes of normal follicular development, atresia of smaller follicles and dominant follicle selection hence induces mono-follicular growth that more closely resembles to a natural menstrual cycle and increases the chance of achieving a singleton pregnancy [10]. Besides these, letrozole has a short half-life of 45 minutes [7]. All these factors make letrozole an attractive option in infertility treatments. Utilization of letrozole to improve IVF outcomes in poor responder women was first proposed by Mitwally, and colleagues [11]. However, preliminary reports involving babies conceived via letrozole administration indicated an increased risk of cardiac and locomotor defects in

Page 2/10 
these infants [12]. Therefore effects of adjuvant letrozole administration on IVF success is a particularly understudied topic. Although not confirmed by larger studies, concerns about increased risk of congenital defects gave rise to a prudent approach to utilization of letrozole for infertility and its use stayed off-label for ovulation induction. However, recently, considering the latest safety data on letrozole, American College of Obstetricians and Gynecologists now recommends letrozole as first line therapy for infertile women with PCOS due to higher clinical pregnancy and live birth rates comparing to clomiphene citrate [13, 14]. Therefore, contemporarily, utilization of letrozole in reproductive medicine is increasing in frequency [7].

Here we conducted this study to evaluate the effects of adjuvant letrozole administration on IVF/ICSI outcomes in POSEIDON group 3 and POSEIDON group 4 expected POR women.

\section{Materials And Methods}

This study is conducted in a university affiliated infertility center (Memorial Atasehir Hospital affiliated with Uskudar University) in Istanbul by retrospective analysis of hospital records. Ethical approval for this study was obtained from Ethical Committee of Uskudar University at 28/04/2021 (Approval number: 61351342/APRIL 2021-84). Study protocol conforms to the "Declaration of Helsinki-Ethical Principles for Medical Research Involving Human Subjects" and the need for consent was omitted by ethical committee due to the retrospective design.

\section{Study population}

Patients with expected POR (POSEIDON group 3 and group 4) that underwent GnRH antagonist cycles with intra-cytoplasmic sperm injection (ICSI) between 2010 and 2020 were analyzed. POSEIDON group 3 consists of women younger than 35 years old with $A M H<1,2$ and $A F C<5$. POSEIDON group 4 consists of women $\geq 35$ years of age and with $A M H<1,2$ and $A F C<5$. Patients with low $(<18 \mathrm{~kg} / \mathrm{m} 2)$ or high ( $>30 \mathrm{~kg} / \mathrm{m} 2)$ body mass indexes (BMI), patients with endocrine co-morbidities (hyperprolactinemia, thyroid dysfunction, diabetes mellitus, Addison disease, congenital adrenal hyperplasia, Cushing syndrome), cycles with any adjuvant administration except from letrozole, patients with uterine anomalies or a history of surgical uterine anomaly correction, patients with male factor infertility due to azoospermia were excluded. Outcomes of the remaining patients with and without adjuvant letrozole administration were evaluated in the study. Expected POR patients (POSEIDON group 3 and group 4) that underwent $\mathrm{GnRH}$ antagonist cycles with adjuvant letrozole administration were included in letrozole group and patients without any adjuvant administration were included in control group. Cycle outcomes of these groups were compared. Subsequently, patients with adjuvant letrozole administration and controls were re-grouped in accordance with being POSEIDON group 3 or POSEIDON group 4 and data were re-analyzed.

\section{Controlled ovarian stimulation and interventions}

Controlled ovarian stimulation was initiated in 2nd day of menstrual cycle. Letrozole (Femara ${ }^{\circledR}$; Novartis Pharmaceuticals Corporation) was administered $2.5 \mathrm{mg}$ twice daily per-orally, starting from the 2 nd day of menstruation for 5 days. Recombinant follicle stimulating hormone ( $\mathrm{rFSH}$, Gonal-F, Merck Serono S.p.A), human menopausal gonadotropin (hMG, Merional, IBSA Institut Biochimique S.A, Menopur® Ferring Pharmaceuticals) or combination of recombinant luteinizing hormone (rLH) and rFSH (Pergoveris, Merck Serono SA) were used for ovarian stimulation. Cycles were monitored for follicular growth with serial transvaginal ultrasounds and serum hormone levels throughout the course of stimulation. Gonadotropin doses were adjusted accordingly with each patient's follicular growth. GnRH antagonist (Cetrotide $0.25 \mathrm{mg}$, Pierre Fabre Medicament Production) injections were initiated to suppress premature LH peak whenever the leading follicle have reached a diameter of 12-14 mm, and continued to the day of oocyte maturation triggering. Oocyte maturation was triggered when at least one follicle has reached a diameter of $18 \mathrm{~mm}$. Either dual-trigger or traditional hcg-only trigger was used for final oocyte maturation triggering. GnRH agonist of $0.2 \mathrm{mg}$ triptorelin acetate, (Gonapeptyl, Ferring Pharmaceuticals) and 250mcg recombinant human chorionic gonadotropin (Ovitrelle, Merck Serono) were administered concomitantly in patients that dual-triggering was performed. $250 \mathrm{mcg}$ recombinant human chorionic gonadotropin (Ovitrelle, Merck Serono) was used alone for oocyte maturation in women with hcgonly triggering. Oocytes were retrieved under transvaginal ultrasound guidance 35-36 hours after oocyte maturation trigger. Fertilization was conducted by intracytoplasmic sperm injection (ICSI). Embryo quality was graded according to The Society for Assisted Reproductive Technology (SART) grading system [15]. Regarding the SART grading system grade 1 embryos are referred 
as good quality embryos, grade 2 embryos are referred as fair quality embryos and grade 3 embryos are referred as poor quality embryos in this study. Day 3 or day 5 embryos are transferred depending on the condition and physician preference. Embryo transfers were conducted via soft embryo transfer catheters under abdominal ultrasonography guidance. A maximum of 2 embryos were transferred in each attempt. Intravaginal progesterone is initiated in every patient with either 200 mg Lutinus twice a day or $\mathbf{2 0 0}$ mg Progestan three times a day (Lutinus vaginal tablets, Ferring Pharmaceuticals; Progestan Soft Capsules, Koçak Farma Pharmaceutical and Chemical Industry Co.) for luteal phase support and continued to the 8th-10th gestational weeks.

\section{Outcome measures}

Primary outcome of this study was determined as live birth delivery rates in POSEIDON group 3 patients, POSEIDON group 4 patients and overall expected POR patients. Secondary outcomes were total dose of required gonadotropins, number of retrieved oocytes, number of metaphase2 (M2) oocytes, oocyte maturation rate (number of M2 oocytes/number of retrieved oocytes), number of obtained 2 pronuclear (2PN) embryos, fertilization rates (number of 2PN embryos/number of M2 oocytes), embryo quality, implantation rates (gestational sacs observed/transferred embryos), clinical pregnancy rate, cycle cancellation rate and miscarriage rates. Outcome parameters were defined in accordance with The International Glossary on Infertility and Fertility Care, 2017 [16].

\section{Statistical analysis}

Statistical analysis were carried out by IBM SPSS statistics 23 (evaluation version). Descriptive statistics were expressed as mean \pm standard deviations for normally distributed data. Categorical variables were expressed as numbers and percentages (\%). Significance of differences between means were evaluated with Student's $T$ test. Categorical variables were evaluated with Pearson's Chi-Squared test or Fisher's exact test. P values $<0.05$ are considered significant.

\section{Results}

Among the eligible subjects, adjuvant letrozole was administered in 488 women that constitute letrozole group, 2564 women were underwent $\mathrm{GnRH}$ antagonist cycles without any adjuvant administration within the selected period of time and included in control group. Eighty-nine women in letrozole group and 739 women in control group were found to be POSEIDON group 3 patients. Three-hundred-ninety-nine women in letrozole group and 1825 controls were found to be POSEIDON group 4 expected PORs.

Mean age, BMI and the method of oocyte maturation triggering in letrozole group and controls were similar among overall expected POR patients. Mean number of previous unsuccessful IVF attempts was significantly higher in letrozole group (2.33 vs. 2.03; $p=0.002$ ). Total dose of required gonadotropins ( $3209.21 \pm 806.28 \mathrm{IU}$ vs. $3692.65 \pm 999.25 \mathrm{IU} ; \mathrm{p}<0.001)$, days of stimulation ( $9.35 \pm 1.51$ vs. $9.87 \pm 1.72 ; p<0.001)$, endometrial thickness $(9.42 \pm 1.83 \mathrm{~mm}$ vs. $10.19 \pm 1.95 \mathrm{~mm} ; p<0.001)$, peak estradiol $(557.41 \pm 474.16 \mathrm{pg} / \mathrm{ml}$ vs. $1014.09 \pm 503.76 \mathrm{pg} / \mathrm{ml} ; \mathrm{p}<0.001)$ and progesterone levels $(0.65 \pm 0.39 \mathrm{ng} / \mathrm{ml}$ vs. $0.68 \pm 0.31 \mathrm{ng} / \mathrm{ml}$; $p=0.022)$, number of retrieved oocytes ( $3.72 \pm 1.82$ vs. $3.97 \pm 1.72 ; p<0.001)$ were significantly lower in letrozole group. Oocyte maturation rates $(0.78 \pm 0.23$ vs. $0.75 \pm 0.27 ; p=0.001)$, number of $2 P N$ embryos $(2.24 \pm 1.37$ vs. $1.87 \pm 1.0 ; p<0.001)$, fertilization rates $(0.82 \pm 0.30$ vs. $0.72 \pm 0.29 ; p<0.001)$ were significantly higher in adjuvant letrozole administered women in comparison to controls. Comparison of baseline characteristics and outcome parameters of expected POR women with and without adjuvant letrozole administration were given in Table 1. 
Table 1

Comparison of baseline characteristics and cycle outcomes of expected POR women with and without adjuvant letrozole administration.

\begin{tabular}{|c|c|c|c|c|}
\hline & & Letrozole Group & Controls & $P$ value \\
\hline \multicolumn{2}{|l|}{ Number of patients } & 488 & 2564 & \\
\hline \multicolumn{2}{|l|}{ Age (years) } & $37.77 \pm 3.85$ & $37.42 \pm 4.73$ & 0.137 \\
\hline \multicolumn{2}{|l|}{ BMI } & $24.74 \pm 3.16$ & $24.91 \pm 2.87$ & 0.413 \\
\hline \multirow[t]{2}{*}{ Oocyte maturation triggering method } & Dual trigger & $177(36.3 \%)$ & $917(35.8 \%)$ & \multirow[t]{2}{*}{0.837} \\
\hline & Hcg-only triggering & $311(63.7 \%)$ & $1647(64.2 \%)$ & \\
\hline \multirow[t]{2}{*}{ Day of embryo transfer } & Day 3 & $336(74.5 \%)$ & $1699(69.5 \%)$ & \multirow[t]{2}{*}{0.031} \\
\hline & Day 5 & $115(25.5 \%)$ & 747 (30.5\%) & \\
\hline \multicolumn{2}{|l|}{ Number of previous IVF attempts } & $2.33 \pm 1.64$ & $2.03 \pm 1.36$ & 0.002 \\
\hline \multicolumn{2}{|l|}{ Required gonadotropin doses (IU) } & $3209.21 \pm 806.28$ & $3692.65 \pm 999.25$ & $<0.001$ \\
\hline \multicolumn{2}{|l|}{ Days of stimulation } & $9.35 \pm 1.51$ & $9.87 \pm 1.72$ & $<0.001$ \\
\hline \multicolumn{2}{|l|}{ Peak estradiol level (pg/ml) } & $557.41 \pm 474.16$ & $1014.09 \pm 503.76$ & $<0.001$ \\
\hline \multicolumn{2}{|l|}{ Peak progesterone level (ng/ml) } & $0.65 \pm 0.39$ & $0.68 \pm 0.31$ & 0.022 \\
\hline \multicolumn{2}{|l|}{ Endometrial thickness (mm) } & $9.42 \pm 1.83$ & $10.19 \pm 1.95$ & $<0.001$ \\
\hline \multicolumn{2}{|l|}{ Cycle cancellations } & $35(7.2 \%)$ & $117(4.6 \%)$ & 0.017 \\
\hline \multicolumn{2}{|l|}{ Number of retrieved oocytes } & $3.72 \pm 1.82$ & $3.97 \pm 1.72$ & $<0.001$ \\
\hline \multicolumn{2}{|c|}{ Maturation rate (M2 oocytes/Retrieved oocytes) } & $0.78 \pm 0.23$ & $0.75 \pm 0.27$ & 0.001 \\
\hline \multicolumn{2}{|l|}{ Number of M2 oocytes } & $2.80 \pm 1.43$ & $2.86 \pm 1.38$ & 0.078 \\
\hline \multicolumn{2}{|l|}{ Number of 2PN embryos } & $2.24 \pm 1.37$ & $1.87 \pm 1.0$ & $<0.001$ \\
\hline \multirow[t]{3}{*}{ Quality of obtained embryos } & Good & $280(61.8 \%)$ & $1492(61 \%)$ & \multirow[t]{3}{*}{0.182} \\
\hline & Fair & $118(26 \%)$ & $715(29.2 \%)$ & \\
\hline & Poor & $55(12.1 \%)$ & $240(9.8 \%)$ & \\
\hline \multicolumn{2}{|l|}{ Number of frozen embryos } & $0.51 \pm 0.72$ & $0.61 \pm 0.75$ & 0.003 \\
\hline \multicolumn{2}{|l|}{ Fertilization rate per cycle } & $0.82 \pm 0.30$ & $0.72 \pm 0.29$ & $<0.001$ \\
\hline \multicolumn{2}{|l|}{ Implantation rate per cycle } & $0.21 \pm 0.37$ & $0.22 \pm 0.37$ & 0.759 \\
\hline \multicolumn{2}{|l|}{ Clinical pregnancy rate } & $134(27.5 \%)$ & $684(26.7 \%)$ & 0.721 \\
\hline \multicolumn{2}{|l|}{ Live birth delivery rate $(n)$} & $17.5 \%(119)$ & $26.7 \%(684)$ & 0.474 \\
\hline \multicolumn{2}{|l|}{ Miscarriage } & $24(17.9 \%)$ & $176(25.7 \%)$ & 0.054 \\
\hline \multicolumn{2}{|l|}{ Multiple pregnancies } & $9(1.8 \%)$ & $79(3.1 \%)$ & 0.081 \\
\hline
\end{tabular}

Analysis revealed no significant differences in terms of $\mathrm{BMI}$, oocyte maturation trigger method and days of embryo transfer in letrozole groups and controls among POSEIDON group 3 and group 4 women. Number of previous IVF attempts were higher in letrozole groups comparing to controls in both POSEIDON group3 and 4 women. Required gonadotropin doses, peak estradiol levels, days of stimulation were all significantly lower in letrozole groups among POSEIDON group 3 and 4 patients. Implantation rates, clinical pregnancy rates and live birth delivery rates were found significantly higher in letrozole group in POSEIDON group 3 women however no significant differences between letrozole group and controls were found in these outcomes among 
POSEIDON group 4 women. Comparison of cycle outcomes of POSEIDON group 3 and group 4 women with and without adjuvant letrozole administration were summarized in Table 2. 
Table 2

Comparison of cycle outcomes of POSEIDON group 3 and group 4 women with and without adjuvant letrozole administration

\begin{tabular}{|c|c|c|c|c|c|c|c|}
\hline & \multicolumn{3}{|c|}{ POSEIDON GROUP 3} & \multicolumn{3}{|c|}{ POSEIDON GROUP 4} \\
\hline & & Letrozole Group & Controls & \multirow{2}{*}{$\begin{array}{l}\mathrm{P} \\
\text { value }\end{array}$} & Letrozole Group & Controls & \multirow{2}{*}{$\begin{array}{l}\mathrm{P} \\
\text { value }\end{array}$} \\
\hline \multicolumn{2}{|c|}{ Number of patients } & 89 & 739 & & 399 & 1825 & \\
\hline \multicolumn{2}{|l|}{ Age (years) } & $31.40 \pm 2.62$ & $31.49 \pm 3.08$ & 0.271 & $39.18 \pm 2.37$ & $39.82 \pm 2.76$ & 0.001 \\
\hline \multicolumn{2}{|l|}{ BMI } & $24.49 \pm 3.03$ & $24.81 \pm 2.85$ & 0.565 & $24.79 \pm 3.19$ & $24.95 \pm 2.88$ & 0.484 \\
\hline \multirow{2}{*}{$\begin{array}{l}\text { Oocyte } \\
\text { maturation } \\
\text { triggering } \\
\text { method }\end{array}$} & $\begin{array}{l}\text { Dual- } \\
\text { trigger }\end{array}$ & $30(33.7 \%)$ & $229(31 \%)$ & \multirow[t]{2}{*}{0.601} & $147(36.3 \%)$ & $688(37.7 \%)$ & \multirow[t]{2}{*}{0.749} \\
\hline & $\begin{array}{l}\text { Hcg-only } \\
\text { triggering }\end{array}$ & $59(66.3 \%)$ & $510(69 \%)$ & & $252(63.2 \%)$ & $1137(62.3 \%)$ & \\
\hline \multirow{2}{*}{$\begin{array}{l}\text { Day of } \\
\text { embryo } \\
\text { transfer }\end{array}$} & Day 3 & $64(75.3 \%)$ & $496(68.5 \%)$ & \multirow[t]{2}{*}{0.2} & $272(74.3 \%)$ & 1203 (69.9\%) & \multirow[t]{2}{*}{0.089} \\
\hline & Day 5 & $21(24.7 \%)$ & $228(30.9 \%)$ & & $94(25.7 \%)$ & $519(30.1 \%)$ & \\
\hline \multicolumn{2}{|c|}{$\begin{array}{l}\text { Number of previous IVF } \\
\text { attempts }\end{array}$} & $2.25 \pm 1.44$ & $1.85 \pm 1.12$ & 0.024 & $2.35 \pm 1.68$ & $2.10 \pm 1.44$ & 0.040 \\
\hline \multicolumn{2}{|c|}{$\begin{array}{l}\text { Required gonadotropin } \\
\text { doses (IU) }\end{array}$} & $2864.65 \pm 878.47$ & $3757.27 \pm 1088.89$ & $<0.001$ & $3286.06 \pm 770.16$ & $3666.48 \pm 959.66$ & $<0.001$ \\
\hline \multicolumn{2}{|c|}{ Days of stimulation } & $9.15 \pm 1.54$ & $9.74 \pm 1.49$ & $<0.001$ & $9.40 \pm 1.51$ & $9.93 \pm 1.80$ & $<0.001$ \\
\hline \multirow{2}{*}{\multicolumn{2}{|c|}{$\begin{array}{l}\text { Peak estradiol level } \\
(\mathrm{pg} / \mathrm{ml})\end{array}$}} & $473.80 \pm 360.45$ & & $<0.001$ & $576.15 \pm 494.42$ & $1021.75 \pm 521.57$ & $<0.001$ \\
\hline & & & $995.20 \pm 456.61$ & & & & \\
\hline \multicolumn{2}{|c|}{$\begin{array}{l}\text { Peak progesterone level } \\
(\mathrm{ng} / \mathrm{ml})\end{array}$} & $0.73 \pm 0.41$ & $0.69 \pm 0.31$ & 0.534 & $0.63 \pm 0.39$ & $0.67 \pm 0.31$ & 0.009 \\
\hline \multicolumn{2}{|c|}{$\begin{array}{l}\text { Endometrial thickness } \\
(\mathrm{mm})\end{array}$} & $9.80 \pm 1.62$ & $10.11 \pm 1.88$ & 0.189 & $9.34 \pm 1.86$ & $10.22 \pm 1.99$ & $<0.001$ \\
\hline \multicolumn{2}{|c|}{ Cycle cancellations } & $4(4.5 \%)$ & $15(2 \%)$ & 0.138 & $31(7.8 \%)$ & $102(5.6 \%)$ & 0.096 \\
\hline \multicolumn{2}{|c|}{$\begin{array}{l}\text { Number of retrieved } \\
\text { oocytes }\end{array}$} & $4.17 \pm 1.87$ & $4.45 \pm 1.74$ & 0.067 & $3.62 \pm 1.80$ & $3.77 \pm 1.67$ & 0.005 \\
\hline \multicolumn{2}{|c|}{$\begin{array}{l}\text { Maturation rate (M2 } \\
\text { oocytes/Retrieved } \\
\text { oocytes) }\end{array}$} & $0.83 \pm 0.19$ & $0.75 \pm 0.36$ & $<0.001$ & $0.77 \pm 0.23$ & $0.75 \pm 0.23$ & 0.077 \\
\hline \multicolumn{2}{|c|}{ Number of M2 oocytes } & $3.28 \pm 1.36$ & $3.18 \pm 1.43$ & 0.719 & $2.69 \pm 1.43$ & $2.73 \pm 1.33$ & 0.116 \\
\hline \multicolumn{2}{|c|}{$\begin{array}{l}\text { Number of } 2 \mathrm{PN} \\
\text { embryos }\end{array}$} & $2.65 \pm 1.41$ & $1.98 \pm 0.86$ & $<0.001$ & $2.15 \pm 1.35$ & $1.83 \pm 1.04$ & $<0.001$ \\
\hline \multirow{3}{*}{$\begin{array}{l}\text { Quality of } \\
\text { obtained } \\
\text { embryos }\end{array}$} & Good & $57(67.1 \%)$ & $446(61.6 \%)$ & \multirow[t]{3}{*}{0.402} & $223(60.6 \%)$ & $1046(60.7 \%)$ & \multirow[t]{3}{*}{0.330} \\
\hline & Fair & $19(22.4 \%)$ & $212(29,3 \%)$ & & $99(26.9 \%)$ & $503(29.2 \%)$ & \\
\hline & Poor & $9(10.6 \%)$ & $66(9.1 \%)$ & & $46(12.5 \%)$ & $174(10.1 \%)$ & \\
\hline \multicolumn{2}{|c|}{$\begin{array}{l}\text { Number of frozen } \\
\text { embryos }\end{array}$} & $0.90 \pm 0.80$ & $0.90 \pm 0.81$ & 0.911 & $0.42 \pm 0.67$ & $0.49 \pm 0.69$ & 0.037 \\
\hline \multicolumn{2}{|c|}{$\begin{array}{l}\text { Fertilization rate per } \\
\text { cycle }\end{array}$} & $0.82 \pm 0.25$ & $0.70 \pm 0.29$ & $<0.001$ & $0.81 \pm 0.30$ & $0.72 \pm 0.29$ & $<0.001$ \\
\hline \multicolumn{2}{|c|}{$\begin{array}{l}\text { Implantation rate per } \\
\text { cycle }\end{array}$} & $0.39 \pm 0.46$ & $0.27 \pm 0.40$ & 0.010 & $0.18 \pm 0.35$ & $0.20 \pm 0.36$ & 0.593 \\
\hline \multicolumn{2}{|c|}{ Clinical pregnancy rate } & $41(46.1 \%)$ & $244(33 \%)$ & 0.014 & $93(23.3 \%)$ & $440(24.1 \%)$ & 0.734 \\
\hline
\end{tabular}




\begin{tabular}{|lllllll|}
\hline $\begin{array}{l}\text { Live birth delivery rate } \\
(\mathbf{n})\end{array}$ & $42.7 \%(38)$ & $31.5 \%(233)$ & 0.034 & $20,3 \%(81)$ & $19.4 \%(354)$ & 0.680 \\
\hline Miscarriages & $7(17.1 \%)$ & $49(20.1 \%)$ & 0.654 & $17(18.3 \%)$ & $127(28.9 \%)$ & 0.037 \\
\hline Multiple pregnancies & $4(4.5 \%)$ & $38(5.1 \%)$ & 0.217 & $5(1.3 \%)$ & $41(2.2 \%)$ & 0.151 \\
\hline
\end{tabular}

\section{Discussion}

This study was conducted to assess the effects of adjuvant letrozole administration on IVF/ICSI outcomes of POSEIDON group 3 and 4 expected POR women. Due to the results obtained, letrozole administration seems to significantly improve implantation rates, clinical pregnancy rates and live birth delivery rates in POSEIDON group 3 patients whereas no significant differences were found in terms of these parameters among POSEIDON group 4 patients.

Studies evaluating the effects of letrozole administration on IVF outcomes in poor responder women have inconsistent results regarding implantation rates, clinical pregnancy rates and live birth rates in literature. Ebrahimi et al. conducted a randomized controlled trial to evaluate the effects of letrozole co-administration on IVF outcomes of poor responders underwent GnRH antagonist protocols [17]. They found no significant differences in implantation rates and clinical pregnancy rates therefore concluded that letrozole administration does not improve IVF outcomes in poor responders. Garcia-Velasco et al. demonstrated an increase in implantation rates with $2.5 \mathrm{mg}$ letrozole administration for first five days of the cycle [18]. Apart from these, most of the studies found increasing trends for clinical pregnancies in letrozole administered poor responder women in comparison to controls without reaching statistical significance [19-22]. Whereas Davar et al observed a decrease in clinical pregnancy rates with letrozole administration [23]. Up to $50 \%$ of IVF failures could be attributed to implantation failure [24]. Implantation rate is a reflection of conjoint effects of both endometrial receptivity and embryo quality. As letrozole blocks aromatase activity, its administration reduces estradiol levels reached following ovarian stimulation and limits deleterious effects of supraphysiological estradiol levels on endometrial receptivity [25]. Furthermore, endometrial integrin expression is a known marker of endometrial receptivity and previously, studies showed significant increase in endometrial integrin expressions with letrozole administration $[26,27]$. However, low embryo quality is a major concern in older women and known as the dominant determinant of implantation rates therefore the clinical pregnancy rates in these patients [26]. Inconsistent findings in previous studies might be results of small sample sizes as well as the criteria used to define or classify poor responder women.

Previous studies demonstrated that letrozole administration reduces required gonadotropin doses by improving follicular sensitivity to FSH stimulation in poor responder women thus cutting the costs of ovarian stimulation $[19,28]$. In our study we found significantly decreased gonadotropin requirements as well as shorter duration of stimulation in both POSEIDON group 3 and group 4 expected POR women that might render the process of ovarian stimulation relatively more patient friendly.

As we mentioned earlier embryo quality is a major determinant in achieving clinical pregnancies in older women. In our study, we observed that $5 \mathrm{mg} /$ day of letrozole administration for five days does not create an improvement in embryo quality in both POSEIDON group 3 and POSEIDON group 4 women. However, Shapira et al. demonstrated that extended use of $5 \mathrm{mg} / \mathrm{day}$ letrozole, starting from the first day of gonadotropin stimulation until the trigger day, increases embryo quality in poor responders [29]. They neither evaluated clinical pregnancy rates and live birth delivery rates in their study nor used the stratification system proposed by POSEIDON study group. However, improved embryo quality could induce an increase in clinical pregnancy rates particularly in POSEIDON group 4 patients. Nevertheless, further studies investigating the effects of extended letrozole administration in POSEIDON group 4 women could elucidate this issue.

In conclusion $5 \mathrm{mg}$ /day of adjuvant letrozole administration for five days appears to increase implantation rates, clinical pregnancy rates and live birth delivery rates in POSEIDON group 3 expected POR women.

\section{Declarations}

Authors' contributions: All authors contributed to the study conception and design. Data collection and analysis were performed by A Kahraman and F Tulek. The first draft of the manuscript was written by A Kahraman and F Tulek. All authors commented on 
previous versions of the manuscript. All authors read and approved the final manuscript.

Conflict of interest: The authors declare that they have no conflict of interest.

Ethical approval: All procedures performed in studies involving human participants were in accordance with the ethical standards of the institutional and/or national research committees and with the 1964 Helsinki Declaration and its later amendments or comparable ethical standards. Ethical approval for this study was obtained from Ethical Committee of Uskudar University at 28/04/2021 (Approval number: 61351342/APRIL 2021-84).

\section{Funding: None}

\section{References}

1. Ovarian Stimulation TEGGO, Bosch E, Broer S et al (2020) ESHRE guideline: ovarian stimulation for IVF/ICSI Hum Reprod Open. ;2020(2):hoaa009. Published 2020 May 1. doi:10.1093/hropen/hoaa009

2. Zhang Y, Zhang C, Shu J et al (2020) Adjuvant treatment strategies in ovarian stimulation for poor responders undergoing IVF: a systematic review and network meta-analysis. Hum Reprod Update 26(2):247-263. doi:10.1093/humupd/dmz046

3. Busnelli A, Papaleo E, Del Prato D et al (2015) A retrospective evaluation of prognosis and cost-effectiveness of IVF in poor responders according to the Bologna criteria. Hum Reprod 30(2):315-322. doi:10.1093/humrep/deu319

4. Chai J, Lee VC, Yeung TW, Li HW, Ho PC, Ng EH (2015) Correction: Live Birth and Cumulative Live Birth Rates in Expected Poor Ovarian Responders Defined by the Bologna Criteria Following IVF/ICSI Treatment. PLoS ONE 10(6):e0131334 Published 2015 Jun 22. doi:10.1371/journal.pone.0131334

5. Humaidan P, Alviggi C, Fischer R, Esteves SC The novel POSEIDON stratification of 'Low prognosis patients in Assisted Reproductive Technology' and its proposed marker of successful outcome. F1000Res. 2016 Dec 23;5:2911. doi: 10.12688/f1000research.10382.1. PMID: 28232864; PMCID: PMC5302217

6. Diminished Ovarian Reserve and Assisted Reproductive (2020) Technologies Current Research and Clinical Management, Orhan Bukulmez. Springer International Publishing

7. Rose BI, Brown SE (2020 Sep) A review of the physiology behind letrozole applications in infertility: are current protocols optimal? J Assist Reprod Genet 37(9):2093-2104. doi: 10.1007/s10815-020-01892-6. Epub 2020 Jul 26. PMID: 32712844; PMCID: PMC7492298

8. Frattarelli JL, Peterson EH (2004) Jun;81(6):1713-4 Effect of androgen levels on in vitro fertilization cycles. Fertil Steril. doi: 10.1016/j.fertnstert.2003.11.032. PMID: 15193506

9. Bosdou JK, Venetis CA, Kolibianakis EM, Toulis KA, Goulis DG, Zepiridis L, Tarlatzis BC (2012) Mar-Apr;18(2):127-45 The use of androgens or androgen-modulating agents in poor responders undergoing in vitro fertilization: a systematic review and meta-analysis. Hum Reprod Update. doi: 10.1093/humupd/dmr051. Epub 2012 Feb 3. PMID: 22307331

10. Kar S (2013 Apr) Current evidence supporting "letrozole" for ovulation induction. J Hum Reprod Sci 6(2):93-98. doi: 10.4103/0974-1208.117166. PMID: 24082649; PMCID: PMC3778612

11. Mitwally MFM, Casper RF (2001) Aromatase inhibition improves ovarian response to FSH: a potential option for low responders during ovarian stimulation. Fertil Steril 754S1:S8-S9

12. Biljan MM, Hemmings R, Brassard N (2005) The outcome of 150 babies following the treatment with letrozole or letrozole and gonadotropins. Fertil Steril 84(Suppl 1):S95

13. American College of Obstetricians and Gynecologists' Committee on Practice Bulletins-Gynecology. ACOG Practice Bulletin No. 194: Polycystic Ovary Syndrome [published correction appears in Obstet Gynecol (2020) Sep;136(3):638] Obstet Gynecol. 2018;131(6):e157-e171. doi:10.1097/AOG.0000000000002656

14. Legro RS, Diamond MP, Coutifaris C, Schlaff WD, Alvero R, Casson P, Christman GM, Rosen RM, Cedars MI, Hansen KR, Robinson R, Baker V, Usadi R, Dodson WC, Estes SJ, Kunselman A, Stetter C, Barnhart KT, Coward RM, Trussell JC, Krawetz SA, Santoro N, Huang H, Zhang H, Eisenberg E ; Reproductive Medicine Network. Pregnancy registry: three-year follow-up of 
children conceived from letrozole, clomiphene, or gonadotropins.Fertil Steril. 2020May; 113(5):1005-1013. doi: 10.1016/j.fertnstert.2019.12.023. PMID: 32386612; PMCID: PMC7376442.

15. Racowsky C, Vernon M, Mayer J et al (2010) Standardization of grading embryo morphology. J Assist Reprod Genet 27(8):437-439. doi:10.1007/s10815-010-9443-2

16. Zegers-Hochschild F, Adamson GD, Dyer S, Racowsky C, de Mouzon J, Sokol R, Rienzi L, Sunde A, Schmidt L, Cooke ID, Simpson JL, van der Poel S (2017) Sep;108(3):393-406 The International Glossary on Infertility and Fertility Care, 2017. Fertil Steril. doi: 10.1016/j.fertnstert.2017.06.005. Epub 2017 Jul 29. PMID: 28760517

17. Ebrahimi M, Akbari-Asbagh F, Ghalandar-Attar M Letrozole+ GnRH antagonist stimulation protocol in poor ovarian responders undergoing intracytoplasmic sperm injection cycles: An RCT.Int J Reprod Biomed. 2017Feb; 15(2):101-108. PMID: 28462402; PMCID: PMC5405223.

18. Garcia-Velasco JA, Moreno L, Pacheco A et al (2005) The aromatase inhibitor letrozole increases the concentration of intraovarian androgens and improves in vitro fertilization outcome in low responder patients: a pilot study. Fertil Steril 84(1):82-87. doi:10.1016/j.fertnstert.2005.01.117

19. Ozmen B, Sönmezer M, Atabekoglu CS, Olmus H (2009) Oct;19(4):478-85 Use of aromatase inhibitors in poor-responder patients receiving GnRH antagonist protocols. Reprod Biomed Online. doi: 10.1016/j.rbmo.2009.05.007. PMID: 19909587

20. Ozcan Cenksoy P, Ficicioglu C, Kizilkale O, Suhha Bostanci M, Bakacak M, Yesiladali M, Kaspar C (2014) The comparision of effect of microdose $\mathrm{GnRH}$-a flare-up, $\mathrm{GnRH}$ antagonist/aromatase inhibitor letrozole and $\mathrm{GnRH}$ antagonist/clomiphene citrate protocols on IVF outcomes in poor responder patients. Gynecol Endocrinol. 2014 Jul;30(7):485-9. doi:

10.3109/09513590.2014.893571. Mar 5. PMID: 24592985

21. Lee KH, Kim CH, Suk HJ, Lee YJ, Kwon SK, Kim SH, Chae HD, Kang BM (2014 May) The effect of aromatase inhibitor letrozole incorporated in gonadotrophin-releasing hormone antagonist multiple dose protocol in poor responders undergoing in vitro fertilization. Obstet Gynecol Sci 57(3):216-222. doi: 10.5468/ogs.2014.57.3.216. Epub 2014 May 15. PMID: 24883293; PMCID: PMC4038688

22. Moini A, Lavasani Z, Kashani L, Mojtahedi MF, Yamini N Letrozole as co-treatment agent in ovarian stimulation antagonist protocol in poor responders: A double-blind randomized clinical trial. Int J Reprod Biomed. 2019 Sep 22;17(9):653-660. doi: 10.18502/ijrm.v17i9.5101. PMID: 31646260; PMCID: PMC6804330

23. Davar R, Oskouian H, Ahmadi S, Firouzabadi RD (2010) Sep;49(3):297-301 GnRH antagonist/letrozole versus microdose $\mathrm{GnRH}$ agonist flare protocol in poor responders undergoing in vitro fertilization. Taiwan J Obstet Gynecol. doi: 10.1016/S1028-4559(10)60064-2. PMID: 21056314

24. de los Santos MJ, Mercader A, Galán A, Albert C, Romero JL, Pellicer A (2003) Implantation rates after two, three, or five days of embryo culture. Placenta. Oct;24 Suppl B:S13-9. doi: 10.1016/s0143-4004(03)00172-3. PMID: 14559025

25. Mitwally MF, Casper RF, Diamond MP The role of aromatase inhibitors in ameliorating deleterious effects of ovarian stimulation on outcome of infertility treatment.Reprod Biol Endocrinol. 2005 Oct4;3:54. doi: 10.1186/1477-7827-3-54. PMID: 16202169; PMCID: PMC1266397.

26. Miller PB, Parnell BA, Bushnell G, Tallman N, Forstein DA, Higdon HL 3rd, Kitawaki J, Lessey BA (2012 Mar) Endometrial receptivity defects during IVF cycles with and without letrozole. Hum Reprod 27(3):881-888. doi: 10.1093/humrep/der452. Epub 2012 Jan 13. PMID: 22246449; PMCID: PMC3279128

27. Ganesh A, Chauhan N, Das S, Chakravarty B, Chaudhury K (2014 Apr) Endometrial receptivity markers in infertile women stimulated with letrozole compared with clomiphene citrate and natural cycles. Syst Biol Reprod Med 60(2):105-111 Epub 2013 Dec 5. PMID: 24304327

28. Yang AM, Cui N, Sun YF, Hao GM (2021) Letrozole for Female Infertility. Front Endocrinol (Lausanne). 16:676133. 10.3389/fendo.2021.676133. PMID: 34220713; PMCID: PMC8245002

29. Shapira M, Orvieto R, Lebovitz O, Nahum R, Aizer A, Segev-Zahav A, Haas J Does daily co administration of gonadotropins and letrozole during the ovarian stimulation improve IVF outcome for poor and sub optimal responders? J Ovarian Res. 2020 Jun8;13(1):66. doi: 10.1186/s13048-020-00666-z. PMID: 32513279; PMCID: PMC7282086. 